\title{
INTEGRATIVE ANALYSES FOR OMICS DATA: A BAYESIAN MIXTURE MODEL TO ASSESS THE CONCORDANCE OF CHIP-CHIP AND CHIP-SEQ MEASUREMENTS
}

Martin Schäfer ${ }^{1}$, Otgonzul Lkhagvasuren ${ }^{1}$, Hans-Ulrich Klein ${ }^{2}$, Christian Elling ${ }^{3}$, Torsten Wüstefeld ${ }^{4,5}$, Carsten Müller-Tidow ${ }^{3}$, Lars Zender ${ }^{4,5}$, Steffen Koschmieder ${ }^{6}$, Martin Dugas $^{2}$, Katja Ickstadt ${ }^{1}$

${ }^{1}$ Department of Statistics, TU Dortmund University, Dortmund, Germany, ${ }^{2}$ Institute of Medical Informatics, University of Münster, Münster, Germany, ${ }^{3}$ Department of Medicine A, University Hospital Münster, Münster, Germany, ${ }^{4}$ Helmholtz Centre for Infection Research, Braunschweig, Germany, ${ }^{5}$ Department of Gastroenterology, Hepatology and Endocrinology, Medical School Hannover, Hannover, Germany, ${ }^{6}$ Internal Medicine IV, University Hospital Aachen, Aachen, Germany

Running head: Integrative analyses for Omics data

Corresponding author:

Martin Schäfer

Department of Statistics, TU Dortmund University, Vogelpothsweg 87, 44221 Dortmund, Germany.

Phone: +49 231755 5918, Fax: +49 2317555303

E-mail: martin.schaefer@tu-dortmund.de

Otgonzul Lkhagvasuren

fax: +492317555303

email: Lkhagvasuren79@gmx.de

Hans-Ulrich Klein

phone: +49 2518358405 , fax: +49 2518355277

email: h.klein@uni-muenster.de

Christian Elling 
phone: +49 25183 47587, fax: +49 2518352673

email: c.elling@web.de

Torsten Wüstefeld

phone: +49 5316181 3130, fax: +49 531 6181-3099

email: Torsten.Wuestefeld@helmholtz-hzi.de

and

phone: +49 5115323732

email: Wuestefeld.Torsten@mh-hannover.de

Carsten Müller-Tidow

phone: +49 25183 56229, fax: +49 2518352673

email: muellerc@uni-muenster.de

Lars Zender

phone: +49 5316181 3130, fax: +49 531 6181-3099

email: Lars.Zender@helmholtz-hzi.de

and

phone: +49 511532 3732, email: Zender.Lars@mh-hannover.de

Steffen Koschmieder

phone: +49 24180 89713, fax: +49 2418082449

email: SKoschmieder@ukaachen.de

\section{Martin Dugas}

phone: +49 25183 55262, fax: +49 2518355277

email: dugas@uni-muenster.de

\section{Katja Ickstadt}

phone: +49 231755 3111, fax: +49 2317555303

email: ickstadt@statistik.tu-dortmund.de 


\begin{abstract}
The analysis of different variations in genomics, transcriptomics, epigenomics and proteomics has increased considerably in recent years. This is especially due to the success of microarray and, more recently, sequencing technology. Apart from understanding mechanisms of disease pathogenesis on a molecular basis, e.g., in cancer research, the challenge of analyzing such different data types in an integrated way has become increasingly important also for the validation of new sequencing technologies with maximum resolution. For this purpose, a methodological framework for their comparison with microarray techniques in the context of smallest sample sizes which result from the high costs of experiments is proposed in this work. Based on an adaptation of the externally centered correlation coefficient (Schäfer et al., 2009), it is demonstrated how a Bayesian mixture model can be applied to compare and classify measurements of histone acetylation that stem from chromatin immunoprecipitation combined with either microarray (ChIP-chip) or sequencing techniques (ChIP-seq) for the identification of DNA fragments. Here, we characterized the murine hematopoietic cell line 32D, which was transduced with the oncogene BCR-ABL, the hallmark of chronic myeloid leukemia. Cells were compared to mock-transduced cells as control. Activation or inhibition of other genes by histone modifications induced by the oncogene are considered important in such a context for the understanding of the disease.
\end{abstract}

\title{
Key words
}

Bayesian analysis, mixture model, microarray, sequencing, high throughput, leukemia, cancer, epigenomics, histone acetylation, chromatin immunoprecipitation 


\section{Introduction}

The areas of biomedical research often summarized as Omics, in particular genomics, transcriptomics, epigenomics and proteomics, have gained importance in recent years due to technological advances facilitating their analysis. Especially the success of microarrays has helped to use Omics data for understanding mechanisms of disease pathogenesis on a molecular basis, e.g., in cancer research. The increasing availability of data has lead to joint, mostly paired, analyses of various data types with the aim of combining information as well as validating results and conclusions based on single Omics data types. One example are joint analyses of copy number and gene expression data, i.e., of genomic and transcriptomic data (see, e.g., Ortiz-Estevez et al., 2011; van Wieringen \& van de Wiel, 2009). The development and increasing importance of nextgeneration sequencing technologies, which in principle provide maximum resolution without restriction to a fixed number of probes at which information is gathered, is creating the need for a further type of joint analyses: Assessing the accuracy and consistency of results from microarray platforms and next-generation sequencing when targeting the same molecular biologic questions.

In epigenomics, modifications of histones - proteins around which the DNA winds - are an important focus of research because such histone modifications may activate or inhibit the expression of genes and analyzing their functioning may thus help to understand the cause of genetic diseases. Chromatin immunoprecipitation (ChIP) is a molecular biologic method to assay histone modifications; in ChIP, specific antibodies are used to select modified histones together with DNA fragments wound around the histones. Then, DNA fragments are isolated from the histones. In order to determine the genomic positions of the modified histones, the genomic positions of the DNA fragments have to be determined. This can be carried out by labelling the DNA fragments with fluorescent dye, hybridizing them to a microarray containing millions of small fixed DNA sequences and deducing the amount of hybridized DNA from intensitiy values measured with a scanner (ChIP-chip, see Mockler \& Ecker, 2005). Alternatively, the DNA fragments can be sequenced directly using next-generation sequencing technologies and compared to a reference genome (ChIP-seq, see Park, 2009).

A number of methods have been introduced for analysis of both ChIP-chip and ChIP- 
seq data (e.g., Mo and Liang, 2010; Kuan et al., 2011), but work on the joint analysis of these two inputs is still essentially missing, with the exception of Ho et al. (2011) who performed a simple correlation analysis for comparison. The values given by microarray and sequencing techniques are not directly comparable since microarrays give continuous intensity values for fixed locations, while sequencing provides positions of bound sequences. It can be counted how many sequences overlap a given location in order to jointly analyze both techniques, but still equal values given by the two methods will not have the same interpretation. While a correlation analysis in principle can be helpful for a comparison in such a situation, it is not the absolute values from both methods that are most meaningful for an interpretation of the results in medical and biological terms, but rather the deviation presented by values obtained from a collective of (e.g., cancer) patients w.r.t. those coming from a normal control collective. Moreover, in sequencing experiments like ChIP-seq, including a control collective is almost mandatory since DNA fragments with a high guanine-cytosine content are sequenced more efficiently than others. These and other effects give rise to artefacts that can be cancelled out by calculating quotients of values from two collectives (Vega et al., 2009).

The simultaneous comparison of two different variables and two collectives has been tackled in bioinformatics literature, at least implicitly by using ratios calculated from two collectives for both variables (see, e.g., Gallegos et al., 2008). Schäfer et al. (2009) address this setting most explicitly and develop an externally centered correlation coefficient for the simultaneous analysis of two data inputs, using the example of gene expression and copy number data, on two different collectives representing cancer patients and normal controls.

In this work, an adaptation of this framework for the comparison of microarray and sequencing results is developed. With the help of Bayesian methodology, we are able to distinguish between loci at which both biotechnologies agree and loci where they disagree. We demonstrate the utility of our approach on a murine cell line which was transduced with BCR-ABL, an oncogene supposedly responsible for a specific subtype of leukemia.

\section{Data}

The data for the comparison were derived from a 32D cell line that was originally 
isolated from murine bone marrow.

At the University Hospital of Münster, 32D cells were retrovirally stably transduced with the oncogene BCR-ABL (Elling et al., 2011), which is the hallmark of chronic myeloid leukemia (CML). Further, 32D cells were mock-transduced with the empty vector and used as a control sample. BCR-ABL is a fusion oncoprotein which arises from the genomic translocation $\mathrm{t}(9 ; 22)$ and can be detected in different human leukemia patients, especially CML and Philadelphia-positive acute lymphoblastic leukemia.

The control cell line was treated with a growth factor in order to make control cells more similar to the cancer cells w.r.t. growth behaviour. Chromatin immunoprecipitation (ChIP) with an antibody against acetylated histone 3 at lysines 9 and 14 (anti$\mathrm{H} 3 \mathrm{~K} 9 \mathrm{Ac} / \mathrm{K} 14 \mathrm{Ac}$ ) was conducted, then the DNA material was amplified by polymerase chain reaction (PCR). The identity of the DNA sequences bound by the antibody was determined separately by ChIP-chip and ChIP-seq methods. While ChIP-chip is based on a fixed number of probes on fixed locations due to microarray design, ChIP-seq is not limited w.r.t. measurement loci and its precision is only limited by the sequencing depth, i.e., the number of matched sequences, defined by the analyst.

In ChIP-seq, the sequences of several million DNA fragments were determined by highthroughput-screening using the Illumina Genome Analyzer IIx. The origin of the short 35 bit pair (bp) sequences was determined by comparing the sequences with the Mus musculus reference genome. In case of our study, if many DNA sequences fall into a certain region, this indicates that the DNA fragments were bound to histones selected by the antibody during ChIP, and hence, the histones were acetylated in this region.

In ChIP-chip, the DNA fragments were labelled with fluorescent dye and hybridized to the Affymetrix GeneChip Mouse Promoter 1.0R array with approximately 4.5 million 25 mer probes covering more than 25000 promotors, i.e., genomic regions near the genes' transcriptional start sites. Subsequently, a scanner measured an intensity value for each probe. The measurement process was repeated three times on the same cells and the mean across the repeated measurements is taken for subsequent analysis.

The resulting data base in this study is thus very small, consisting of only two cell lines, with measurements taken by two different methods each.

\section{Preprocessing}

The ChIP-chip data were preprocessed using the R package Starr (Zacher et al., 2010), 
in particular by performing quantile normalization (Bolstad et al., 2003). For ChIP-seq data, we could align 5,452,964 $(3,666,697)$ reads obtained from the BCR-ABL (control) sample to the reference. Duplicate sequences are likely artefacts from PCR and were removed, reducing the number of reads to 5,111,088 and 3,398,454, respectively. We then randomly deleted further reads in the BCR-ABL sample to get an equal number of reads there compared to the control sample in each chromosome and thus mimick equal sequencing depths. Sequences were aligned by the Burrows-Wheeler Alignment tool (Li \& Durbin, 2009) using Mus musculus NCBI m37 as reference genome.

To match the ChIP-chip and ChIP-seq values, any ChIP-chip intensity value is assigned the amount of ChIP-seq sequences overlapping its probe, producing a continuous intensity value and an integer number per locus. For this purpose, we artificially extended the sequences towards the $3^{\prime}$ end to a total length of $200 \mathrm{bp}$. This results in more overlapping sequences and the choice of $200 \mathrm{bp}$ seems appropriate, since the length of the original DNA fragments in our experimental set-up was between 100bp and $1000 \mathrm{bp}$ with a mean length of at least $200 \mathrm{bp}$, but only the first $35 \mathrm{bp}$ at the 5 ' end could be sequenced due to technical limitations of the current sequencing technology. Still, the matching comes at the cost of excluding some of the sequences from the analysis. Furthermore, sequencing depth in this study was rather small. The loci for which the ChIP-seq value is zero for both the BCR-ABL and the control cell line are therefore omitted for subsequent analysis since it is uncertain whether the absence of bound sequences is due to absence of acetylation or, rather, due to lack of sequencing depth. To avoid the potential interpretative problems connected to these experimental conditions, it appears acceptable to omit some loci. Due to memory limitations in fitting our Bayesian model with standard software, we restrict our analysis to chromosome 9 where in preliminary analyses a high number of overlapping sequences had been identified. This results in a total amount of 104672 loci.

\section{Adaptation of externally centered correlation coefficient}

Schäfer et al. (2009) presented an externally centered correlation coefficient for the analysis of two sources of genetic variation on two different collectives, using gene expression and copy number data as example inputs. Specifically, the coefficient assesses the degree to which abnormalities are present in a cancer collective w.r.t. a 
control collective that are equally directed in the two data sources. Here, we propose to use this idea in a different setting where again two different collectives are given, but rather two techniques for measuring the same type of variation in Omics data are analysed instead of two different types of genetic variation. For this end, adaptations have to be made that are described in the following.

The externally centered correlation coefficient for two random vectors $X=\left(X_{1}, \ldots, X_{m}\right)$ and $Y=\left(Y_{1}, \ldots, Y_{m}\right)$, corresponding to two sets of measurements on $m$ subjects, is defined as follows (Schäfer et al., 2009):

$$
r_{E C}=\frac{\sum_{k=1}^{m}\left(X_{k}-A\right)\left(Y_{k}-B\right)}{\sqrt{\sum_{k=1}^{m}\left(X_{k}-A\right)^{2}} \sqrt{\sum_{k=1}^{m}\left(Y_{k}-B\right)^{2}}},
$$

where $A$ and $B$ are median values across reference samples, e.g., normal controls, for the two types of variation at the investigated locus. All measurements are on the $\log _{2}$-scale. The measure $r_{E C}$ cannot be applied directly to the comparison of the ChIP-chip and ChIP-seq data in this study since for each technology only one sample per biological condition is available $(m=1)$, and so, no type of correlation can be calculated. However, the rectangles defined by the summands in the nominator, used by Schäfer et al. (2009) to construct a Wilcoxon test, remain well interpretable. We propose to employ them as a base measure for analysis; for their standardization a measure of dispersion is used not across samples, but instead across a window of fixed size around each locus $i$, containing loci $l=1, \ldots, n_{i}$, respectively. Since the loci in each window may be quite different in characteristics and also their numbers may vary considerably, we suggest to use a robust measure here that is less sensitive to such differences. A modified measure for the loci $i=1, \ldots, n$ inspired by the externally centered correlation coefficient can then be defined as

$$
Z_{i}=\frac{\left(X_{i}-A_{i}\right)}{\operatorname{med}\left(\left|X_{l}-A_{l}\right|\right)} \frac{\left(Y_{i}-B_{i}\right)}{\operatorname{med}\left(\left|Y_{l}-B_{l}\right|\right)}
$$

$X_{i}$ and $Y_{i}$ are the random variables for the BCR-ABL cell line in ChIP-chip and in ChIP-seq at locus $i$, while $A_{i}$ and $B_{i}$ are the random variables for the corresponding locus $i$ in the control cell line in ChIP-chip and in ChIP-seq.

$Z_{i}, i=1, \ldots, n$, are considered to be identically distributed as a random variable $Z$. In case of presence of abnormalities in the BCR-ABL cell line w.r.t. the control cell line which 
are equally directed in ChIP-chip and ChIP-seq, the distribution of $Z$ is expected to be slanted towards positive values. A distribution with mostly negative values indicates a non-agreement of ChIP-chip and ChIP-seq values.

\section{Normal Bayesian mixture model}

Bayesian normal mixture models have been used in microarray analysis for classifying genes into categories w.r.t., e.g., differential expression, being the target of a transcription factor or not, as well as for classifying copy number measurements into a number of distinct categories, like normal, loss and gain, representing each category by one of the mixing components (Bröet \& Richardson, 2006, Wei \& Pan, 2008). In these works, different input measures $Z$ have been analyzed by mixture models, e.g., log-ratio measurements or transformed p-values. Here, we propose to use the measure (1) inspired by the externally centered correlation coefficient and in general follow the framework presented by Wei and Pan (2008) for their standard normal mixture model. Our goal is to classify the loci such that positive $Z$ values can be discriminated from negative ones as well as zero values and values that are near enough to zero to be explained by random variability. Based on such a classification, the reproducibility of results obtained by ChIP-chip technology by those obtained by ChIP-seq technology (and vice versa) can be judged.

$Z$ is assumed to be a random variable and $Z_{1}, \ldots, Z_{n}$ to be an i.i.d. random sample of $Z$. $T_{1}, \ldots, T_{n}$ are the classifications for all loci $i=1, \ldots, n$. Then the normal mixture model is defined by the joint distribution as follows:

$$
f\left(z_{i}\right)=\sum_{j=1}^{J} \pi_{j} g\left(z_{i} ; \mu_{j}, \sigma_{j}^{2}\right) \text { with } \sum_{j=1}^{J} \pi_{j}=1, \quad i=1, \ldots, N,
$$

where $\pi_{j}$ is the probability of the $j$ th group, $j=1, \ldots, J$, and $g\left(z_{i} ; \mu_{j}, \sigma_{j}^{2}\right)$ is the probability density function of a normal distribution with mean $\mu_{j}$ and variance $\sigma_{j}^{2}$, representing the $j$ th group. $\pi_{j}, \mu_{j}$ and $\sigma_{j}^{2}$ are unknown and estimated by the model.

If every $Z_{i}$ would be represented by a collective of observations, then classical frequentist inference about the $Z_{i}$ would be possible. Since this, however, is not the case and each $Z_{i}$ is represented by only one observation $z_{i}$, the Bayesian framework comes to be of aid.

\section{Prior distributions}


For comparing ChIP-chip and ChIP-seq methods, we employ eight components, three of which we earmark for representing equally directed deviations between ChIP-chip and ChIP-seq measurements for the BCR-ABL cell line compared to those for the control cell line. We employ three further components to represent unequally directed deviations of this kind, while the remaining two components are thought to represent the $Z$ values which are either exactly zero or small enough in their absolute value to not significantly differ from zero. Preliminary analyses showed that the model needs the flexibility provided by the five additional components compared to the setting in Wei and Pan (2006) to achieve the desired classification. The additional component for zero values is needed because after removing loci without any mapped sequence in the ChIPseq data, a considerable proportion of $Z$ values, $12.16 \%$, is still exactly zero. The additional four components for positive and negative values become necessary because the distribution of the $Z$ values has both notably longer and thicker tails than it has in Wei and Pan (2006). However, for interpretation purposes the components can be grouped into three classes (positive/negative/zero or near zero). In cases where the range of $Z$ values is even greater than in our study, more additional components may be necessary. In the following we describe the specification of prior distributions for the model parameters.

Let $T_{i}=1,2,3$ define the groups representing negative values of $Z, T_{i}=6,7,8$ the groups representing positive values of $Z$ and $T_{i}=4,5$ representing $Z$ values equal or near to zero. For the parameters of the corresponding mixing components, we set up prior distributions inspired by those used in Wei and Pan (2008) and Bröet et al. (2006), but choose more informative priors for the means to better achieve the classification of positive and negative $Z$ values. Specifically, for $j=1,2,3$, we set $\mu_{j} \sim N(-10,1) I(a, 0)$, a truncated normal distribution between $a=\min _{i}\left(z_{i}\right)$ and $0 ; \mu_{j} \sim N(10,1) I(0, b)$ with $b=\max _{i}\left(z_{i}\right)$ for $j=6,7,8$, and fix $\mu_{j}=0$ for $j=4,5$. Like in Wei and Pan (2008), the truncated normals are constructed to ensure unique labelling of the normal mixing components. For all eight groups, $j=1, \ldots, 8$, we use $\sigma_{j}^{2} \sim$ Inverse $\operatorname{Gamma}(0.1,0.1)$ and $\left(\pi_{1}, \pi_{2}, \pi_{3}, \pi_{4}, \pi_{5}, \pi_{6}, \pi_{7}, \pi_{8}\right) \sim \operatorname{Dirichlet}(1,1,1,1,1,1,1,1)$.

\section{Results}

To fit the model, we use Markov Chain Monte Carlo (MCMC) methods implemented in 
the standard software WinBUGS (Spiegelhalter et al., 2003). The corresponding code can be obtained from the authors upon request. For all other types of calculations and analyses, we use the statistical software environment R, version 2.14.1 (R Development Core Team, 2011).

Since prior to ChIP, the DNA is cut into pieces of up to at least $1000 \mathrm{bp}$ in size and a maximum of approximately $150 \mathrm{bp}$ wraps around one histone, DNA fragments enriched by the ChIP procedure, i.e., an increased amount of DNA fragments bound to modified histones, can be found at up to roughly $900 \mathrm{bp}$ distance from an acetylated histone. Thus, we choose a window size of $1800 \mathrm{bp}$ for standardization in (1). Non-valid $Z$ values due to, e.g., the denominator being equal to zero in the ChIP-seq part of equation (1), are removed from the analysis.

The posterior distribution of the model parameters is obtained via MCMC methods based on 500 posterior samples, using every 150th iteration out of 75000 with a burn-in of 2000 iterations. The performance of the MCMC algorithm w.r.t convergence and mixing was assessed by examination of trace plots, burn-in and thinning were chosen to ensure that the analysed sample stems from the desired posterior distribution. On an Intel Core i5 64-bit computer with 8 GB RAM, the analysis ran approximately 20 hours. The mean posterior estimates for all model parameters, their standard deviations as well as the number of assigned loci are given in Table 1. In Figure 1, the overall fit of the model to the data can be seen. The visible peaks at -1 and 1 arise due to a certain number of loci for which no other loci fall into their standardizing window, which results in nominator and deminator being equal in terms of absolute value. As intended, components 1 to 3 and components 6 to 8 represent the negative and positive $Z$ values, respectively, and component 5 represents the zero values. Component 4 comes to capture values that are close to zero.

[ Table 1 about here. ]

Loci are assigned to components by their median a posteriori $T_{i}$ values. The implicit classification obtained by the model is visualized in Figure 2 where it can be seen that the model achieves a good separation between the positive, negative and zero $Z$ values, with two extreme negative values sticking out as misclassified which can however be neglected in the overall interpretation.

This makes it possible to do sensible inference about the proportions of positive and 
negative $Z$ values, which is a central goal of the analysis. In the model, for the mixing proportions of the negative components we estimate $\sum_{i=1}^{3} \hat{\pi}_{i}=0.073$, whereas for the mixing proportions of the positive components we estimate $\sum_{i=6}^{8} \hat{\pi}_{i}=0.131$. The latter proportion is nearly twice as big as the first one, and, more importantly, the $95 \%$ credible intervals for $\sum_{i=1}^{3} \hat{\pi}_{i}$ and $\sum_{i=6}^{8} \hat{\pi}_{i}$ are $[0.063,0.086]$ and $[0.102,0.164]$, respectively, i.e., the sums of the mixing proportions of positive components vs. negative components differ significantly from each other and from zero. Thus, the analysis based on the chosen model indicates that ChIP-chip and ChIP-seq technologies agree more than they disagree, although in particular the relatively broad credible interval for $\sum_{i=6}^{8} \hat{\pi}_{i}$ leaves some uncertainty to the question of how much larger the fraction of agreeing measurements is.

The sum of the mixing proportions of the zero components is quite high with $\sum_{i=4,5} \hat{\pi}_{i}=$ $0.794(95 \%$ credible interval $[0.759,0.833])$. Given the unimodal and near-symmetric form of the distribution, this comes not as a surprise. In a case of a more asymmetric or multimodal distribution, more values should be expected to differ notably from zero.

[ Figure 1 about here. ]

[ Figure 2 about here. ]

The proportions of loci classified as belonging to the positive (0.074) and negative components $(0.053)$ are smaller than the estimated mixing proportions of the model because notable parts of the distributions of components 3 and 6 are masked by the distribution of component 4 . The proportion of loci classified as belonging to one of the positive components is greater than the proportion of loci classified as belonging to one of the negative components, but to a smaller extent than is reflected by the corresponding mixing proportions. Again, this suggests caution w.r.t. the question of how much larger the fraction of concordances between ChIP-seq and ChIP-chip is compared to the fraction of discordances.

Our analysis demonstrates the utility of Bayesian methodology in comparisons between 
two sources of data in case of small samples sizes. Model-based estimates as well as classification results help to interpret the distribution of $Z$ values.

The implications of the results are moderately positive for biomedical researchers interested in consistency of important measurement methodologies. Discrepancies of ChIP-seq and ChIP-chip results occur at an amount of loci which is undesirably high compared to the amount of loci classified as showing agreement between the two technologies, and reasons are of interest. We observe a Pearson correlation coefficient of 0.041 in average on chromosome 9, measured across loci within $1 \mathrm{~kb}$ bins, excluding bins containing no sequences in one of the cell lines. Thus, the results based on the $Z$ values reflect to some extent other assessments of association carried out on the ChIPseq and ChIP-chip profiles.

\section{Discussion}

In this analysis we have compared the results obtained from ChIP-chip and ChIP-seq technologies, investigating histone modifications in a murine cell line into which the cancer gene BCR-ABL was transduced, compared to a normal control cell line. In addition to correlation methods which have been used for such kind of analyses, we have adapted the externally centered correlation coefficient (Schäfer et al., 2009) to capture the degree of concordance between the two technologies by an adequate measure, and subsequently applied a Bayesian mixture model to classify loci as presenting 'cancer-reference' value differences that are concordant or discordant between ChIP-chip and ChIP-seq measurements. The mixing proportions of the model components representing either concordant or discordant differences total around only $20 \%$, while most values are estimated to be zero or near enough to zero to be explained by random variability. This may be due to the fact that the same antibody (anti$\mathrm{H} 3 \mathrm{~K} 9 \mathrm{Ac} / \mathrm{K} 14 \mathrm{Ac}$ ) was used for both the BCR-ABL and the control cell line, possibly leading to overly similar ChIP-seq profiles in both.

The sum of the mixing proportions of the model components representing concordant differences $(0.131)$ is bigger than the sum of the mixing proportions of the model components representing discordant differences (0.073), i.e., in our analysis, ChIP-chip and ChIP-seq agree more often than they disagree. The credible intervals of the two sums of proportions suggest that they are significantly different from each other and from zero, but that the real difference between them might be smaller than suggested by 
the point estimates, which is why they should be interpreted with caution. From a practical viewpoint, it would be desirable that the proportion of differences judged as being discordant between ChIP-chip and ChIP-seq be smaller than indicated by the model, ideally not statistically different from zero. The considerable amount of discordances remains disturbing to researchers relying on validity of results.

However, for a number of reasons it can be questioned whether the results can be generalized w.r.t the amount of discordances. First, the control cell line probes were originally treated differently (with a growth factor for ChIP-seq, without a growth factor for ChIP-chip). Since different experimental conditions harm comparability, several months later new ChIP-chip profiles were generated, this time with a growth factor as well. The time between the two measurements may possibly have caused batch effects, whose impact in histone modification analyses has not yet been studied. The growth factor, on the other hand, while intended to increase comparability of the cell lines w.r.t. growth behaviour, may possibly also cause changes w.r.t. other cell characteristics. Also, the sequencing depth was rather small, which makes it likely that a number of low- and medium-size peaks in the sequencing data may not have been detected. Finally, as suggested by Ho et al. (2011), the cross-platform validity of ChIP-chip and ChIP-seq measurements depends heavily on the used antibody, and precisely for anti-H3K9Ac, an antibody similar to the one used in our experiments, they report the worst results of all across several antibodies. In conclusion, it is possible that the experimental conditions have harmed the results in our study. One likely source of bias, the choice of an algorithm to perform peak calling on the ChIP-seq data (see again Ho et al., 2011), was, however, eliminated in our analysis since we did not carry out peak calling. The results indicate that researchers should remain cautious at the moment w.r.t. the mutual reproducibility of ChIP-chip and ChIP-seq results.

The measure used here for assessing the concordance between ChIP-chip and ChIP-seq technologies can be readily adapted to take into account asymmetry w.r.t the two possible types of discordance between the two data types, i.e., cases where the ChIPchip value presents a positive deviation w.r.t the control collective and the ChIP-seq value a negative one should not be treated equal to the reverse case.

We demonstrated the utility of the presented methodological framework for jointly analyzing two data sources in presence of very small sample sizes, independently from the actual results. At least with minor adaptations w.r.t. the number of components and 
prior distributions of their means, this approach comes of aid whenever values are to be classified in a joint analysis of data types in presence of very small sample sizes and common frequentist alternatives fail.

\section{FUNDING}

MS and KI are supported by the Deutsche Forschungsgemeinschaft (Research Training Group Statistical Modeling). HUK and MD are supported by the José Carreras Foundation (DJCLS 09/04). 


\section{REFERENCES}

Bolstad, B. M., Irizarry, R. A., Åstrand, M., and Speed, T. P. 2003. A comparison of normalization methods for high density oligonucleotide array data based on variance and bias. Bioinformatics 19:185-193.

Bröet, P., and Richardson, S. 2006. Detection of gene copy number changes in CGH microarrays using a spatially correlated mixture model. Bioinformatics 22:911-918.

Elling, C., Erben, P., Walz, C., Frickenhaus, M., Schemionek, M., Stehling, M., Serve, H., Cross, N. C. P., Hochhaus, A., Hofmann, W.-K., Berdel, W. E., Müller-Tidow, C., Reiter, A., and Koschmieder, S. 2011. Novel imatinib-sensitive PDGFRA-activating point mutations in hypereosinophilic syndrome induce growth factor independence and leukemia-like disease. Blood 117:2935-2943.

Gallegos R., Mariëlle I., Floor, K., Roepman, P., Rodriguez, J. A., Meijer, G. A., Mooi, W. J., Jassem, E., Niklinski, J., Muley, T., van Zandwijk, N., Smit, E. F., Beebe, K., Neckers, L., Ylstra, B., and Giaccone, G. 2008. Integration of gene dosage and gene expression in non-small cell lung cancer, identification of HSP90 as potential target. PLOS ONE 3(3):e0001722.

Ho, W. K. J., Bishop, E., Karchenko, P. V., Negré, N., White, K. P. and Park, P. J. 2011. ChIP-chip versus ChIP-seq. Lessons for experimental design and data analysis. BMC Genomics 12:134.

Kuan, P. F., Chung, D., Pan, G., Thomson, J. A., Stewart, R., and Keleş, S. 2011. A statistical framework for the analysis of ChIP-seq data. J. Am. Statist. Assoc. 106(495):891-903.

Li, H., and Durbin, R. 2009. Fast and accurate short read alignment with BurrowsWheeler transform. Bioinformatics 25:1754-1760.

Lkhagvasuren, O. 2010. Integrierte Analyse von ChIP-Chip und ChIP-Seq Daten. Bachelor thesis, Department of Statistics, TU Dortmund University. 
Mo, Q., and Liang, F. 2010. Bayesian modeling of ChIP-chip data through a high-order Ising model. Biometrics 66:1284-1294.

Mockler, T. C., and Ecker, J. R. 2005. Applications of DNA tiling arrays for wholegenome analysis. Genomics 85:1-15.

Ortiz-Estevez, M., De las Rivas, J., Fontanillo, C., and Rubio, A. 2011. Segmentation of genomic and transcriptomic microarrays data reveals major correlation between DNA copy number aberrations and gene-loci expression. Genomics 97:86-93.

Park, P. J. 2009. ChIP-seq:advantages and challenges of a maturing technology. Nat. Rev. Genet. 10:669-680.

R Developent Core Team. 2011. R: A language and environment for statistical computing”. R foundation for Statistical Computing, Vienna, Austria. ISBN 3-90005107-0, URL http://www.R-project.org.

Schäfer, M., Schwender, H., Merk, S., Haferlach, C., Ickstadt, K., and Dugas, M. 2009. Integrated analysis of copy number alterations gene expression: a bivariate assessment of equally directed abnormalities. Bioinformatics 25:3228-3235.

Spiegelhalter, D., Thomas, A., Best, N., Lunn, D. 2003. WinBUGS User Manual, Version 1.4. Available at http://www.mrc-bsu.cam.ac.uk/bugs/winbugs/manual14.pdf (as accessed on August 23rd, 2011).

van Wieringen W. N., and van de Wiel M. A. 2009. Nonparametric testing for DNA copy number induced differential mRNA gene expression. Biometrics 65:19-29.

Vega, V. B., Cheung, E., Palanisamy, N. and Sung, W.-K. 2009. Inherent Signals in sequencing-based Chromatin-ImmunoPrecipitation control libraries. PLoS One 4:e5241.

Wei, P., and Pan, W. 2008. Incorporating gene networks into statistical tests for genomic data via a spatially correlated mixture model. Bioinformatics 24:404-411.

Zacher, B., Kuan, P. F., and Tresch, A. 2010. Starr: Simple Tiling ARRay analysis of Affymetrix ChIP-chip data. BMC Bioinformatics 11:194. doi:10.1186/1471-2105-11194. 
Legends to the Tables

TABLE 1. Mean posterior estimates of model parameters (with their standard deviations) and numbers of assigned loci. 
TABLE 1. Mean posterior estimates of model parameters (with their standard deviations) and numbers of assigned loci.

\begin{tabular}{|c|c|c|c|c|c|c|c|c|}
\hline & \multicolumn{8}{|c|}{ Model components } \\
\hline & \multicolumn{3}{|c|}{ negative } & \multicolumn{2}{|c|}{ zero and near zero } & \multicolumn{3}{|c|}{ positive } \\
\hline & 1 & 2 & 3 & 4 & 5 & 6 & 7 & 8 \\
\hline$\mu$ & $\begin{array}{l}-9.531 \\
(1.047)\end{array}$ & $\begin{array}{l}-6.468 \\
(0.725)\end{array}$ & $\begin{array}{l}-3.142 \\
(0.155)\end{array}$ & 0 & 0 & $\begin{array}{l}2.369 \\
(0.303)\end{array}$ & $\begin{array}{l}7.610 \\
(0.732)\end{array}$ & $\begin{array}{l}8.939 \\
(1.207)\end{array}$ \\
\hline$\sigma^{2}$ & $\begin{array}{l}75.026 \\
(37.103)\end{array}$ & $\begin{array}{l}8.216 \\
(2.222)\end{array}$ & $\begin{array}{l}2.580 \\
(0.481)\end{array}$ & $\begin{array}{l}1.677 \\
(0.039)\end{array}$ & $\begin{array}{l}0.00002 \\
(0.0000002)\end{array}$ & $\begin{array}{l}4.711 \\
(0.628)\end{array}$ & $\begin{array}{l}12.533 \\
(2.868)\end{array}$ & $\begin{array}{l}217.066 \\
(93.126)\end{array}$ \\
\hline$\pi$ & $\begin{array}{l}0.003 \\
(0.001)\end{array}$ & $\begin{array}{l}0.015 \\
(0.004)\end{array}$ & $\begin{array}{l}0.056 \\
(0.008)\end{array}$ & $\begin{array}{l}0.671 \\
(0.019)\end{array}$ & $\begin{array}{l}0.124 \\
(0.001)\end{array}$ & $\begin{array}{l}0.117 \\
(0.017)\end{array}$ & $\begin{array}{l}0.012 \\
(0.003)\end{array}$ & $\begin{array}{l}0.002 \\
(0.001)\end{array}$ \\
\hline$\sum \hat{\pi}_{i}$ & \multicolumn{3}{|c|}{$\begin{array}{c}0.073 \\
(0.006)\end{array}$} & \multicolumn{2}{|c|}{$\begin{array}{c}0.795 \\
(0.019)\end{array}$} & \multicolumn{3}{|c|}{$\begin{array}{c}0.131 \\
(0.016)\end{array}$} \\
\hline \multirow{2}{*}{$\begin{array}{l}\text { number of } \\
\text { assigned loci }\end{array}$} & 185 & 980 & 4367 & 78064 & 13278 & 6949 & 763 & 86 \\
\hline & \multicolumn{3}{|c|}{5532} & \multicolumn{2}{|c|}{91342} & \multicolumn{3}{|c|}{7798} \\
\hline
\end{tabular}

